\title{
Chiral effective field theory for nuclear forces: Achievements and challenges
}

\author{
R. Machleidt ${ }^{1, a}$ \\ ${ }^{1}$ Department of Physics, University of Idaho, Moscow, Idaho 83844, USA
}

\begin{abstract}
I start with a historical review of the theories of nuclear forces and then shift to the main focus, which is the chiral effective field theory approach to nuclear forces. I summarize the current status of this approach and discuss the most important open issues: the proper renormalization of the chiral two-nucleon potential and sub-leading three-nucleon forces.
\end{abstract}

\section{Introduction and overview}

The theory of nuclear forces has a long history (cf. Table 1). Based upon the seminal idea by Yukawa [1], first field-theoretic attempts to derive the nucleon-nucleon $(N N)$ interaction focused on pion exchange. While the one-pion exchange turned out to be very useful in explaining $N N$ scattering data and, in particular, the properties of the deuteron [2], multi-pion exchange was beset with serious ambiguities [3] that could not be resolved in a satisfactory way. Thus, the "pion theories" of the 1950s are generally judged as failures-for reasons we understand today: pion dynamics is constrained by chiral symmetry, a crucial point that was unknown in the 1950s.

Historically, the experimental discovery of heavy mesons in the early 1960 s saved the situation. The one-boson-exchange (OBE) model emerged, which still today is the most economical and quantitative phenomenology for describing the $N N$ interaction [4]. The weak point of this model, however, is the scalar-isoscalar "sigma" or "epsilon" boson, for which empirical evidence remains controversial. Since this boson is associated with the correlated (or resonant) exchange of two pions, a vast theoretical effort was launched to derive the $2 \pi$-exchange contribution of the nuclear force, which creates the intermediate range attraction. During this endeavor, which occupied more than a decade, dispersion theory (Stony Brook and Paris potentials) as well as field theory (Partovi-Lomon model, Bonn potential $[4,5])$ were invoked.

The nuclear force problem appeared to be solved; however, with the discovery of quantum chromo-dynamics (QCD), all "meson theories" were relegated to the status of models and the attempts to derive the nuclear force had to start all over again.

The problem with a derivation of nuclear forces from QCD is that this theory is non-perturbative in the low-energy regime characteristic of nuclear physics, which makes direct solutions very difficult. Therefore, during the first round of new attempts, QCD-inspired quark models became popular. The positive aspect of these models is that they try to explain hadron structure and hadron-hadron interactions on an equal footing and, indeed, some of the gross features of the $N N$ interaction are explained

a e-mail: machleid@uidaho.edu 
EPJ Web of Conferences

Table 1. The Theory of Nuclear Forces: Eight Decades of Struggle

\begin{tabular}{|c|c|}
\hline 1935 & Yukawa: Meson Theory \\
\hline 1950's & $\begin{array}{l}\text { The "Pion Theories". } \\
\text { One-pion exchange: good; } \\
\text { Multi-pion exchange: disaster. }\end{array}$ \\
\hline 1960's & $\begin{array}{l}\text { Many pions } \equiv \text { multi-pion resonances: } \sigma, \rho, \omega, \ldots \\
\text { The One-Boson-Exchange Model: success. }\end{array}$ \\
\hline 1970's & $\begin{array}{l}\text { Refinement of meson theory: Sophisticated } 2 \pi \text { exchange models; } \\
\text { Partovi-Lomon, Stony Brook, Paris, Bonn. }\end{array}$ \\
\hline 1980's & $\begin{array}{l}\text { Nuclear physicists discover QCD: } \\
\text { Quark Models. }\end{array}$ \\
\hline $\begin{array}{l}\text { 1990's } \\
\text { and beyond }\end{array}$ & $\begin{array}{c}\text { Nuclear physicists discover EFT; Weinberg, van Kolck, ... } \\
\text { Back to Yukawa's Meson (Pion) Theory! } \\
\text { But, constrained by Chiral Symmetry. }\end{array}$ \\
\hline
\end{tabular}

successfully. However, on a critical note, it must be pointed out that these quark-based approaches are nothing but another set of models and, thus, do not represent fundamental progress. For the purpose of describing hadron-hadron interactions, one may equally well stay with the simpler and much more quantitative meson models.

A major breakthrough occurred when the concept of an effective field theory (EFT) was introduced and applied to low-energy QCD. As outlined by Weinberg in a seminal paper [6], one has to write down the most general Lagrangian consistent with the assumed symmetry principles, particularly the (broken) chiral symmetry of QCD. At low energy, the effective degrees of freedom are pions (the Goldstone bosons of the broken symmetry) and nucleons rather than quarks and gluons; heavy mesons and nucleon resonances are integrated out. So, the circle of history is closing and we are back to Yukawa's meson (pion) theory, except that we have finally learned how to deal with it: broken chiral symmetry is a crucial constraint that generates and controls the dynamics and establishes a clear connection with the underlying theory, QCD.

Chiral EFT allows for a systematic low-momentum expansion known as chiral perturbation theory (ChPT) [6]. Contributions are analyzed in terms of powers of small momenta over the large scale: $\left(Q / \Lambda_{\chi}\right)^{v}$, where $Q$ is generic for a momentum (nucleon three-momentum or pion four-momentum) or pion mass and $\Lambda_{\chi} \approx 1 \mathrm{GeV}$ is the chiral symmetry breaking scale. In terms of this low-momentum expansion, nuclear forces emerge as a hierarchy (Fig. 1).

The past 15 years have seen great progress in developing nuclear forces by moving through this hierarchy [7-14]. As a result, nucleon-nucleon $(N N)$ potentials of high precision have been constructed, which are based upon ChPT carried to next-to-next-to-next-to-leading order ( $\mathrm{N}^{3} \mathrm{LO}$ ) [12-14] ( cf. Table 2).

Chiral two-nucleon forces have been used in many microscopic calculations of nuclear reactions and structure [17-21] and the combination of chiral two- and three-nucleon forces has been applied in few-nucleon reactions [22-26], structure of light- and medium-mass nuclei [27-35], and nuclear and 
INPC 2013

\section{N Force \\ 3N Force \\ 4N Force}
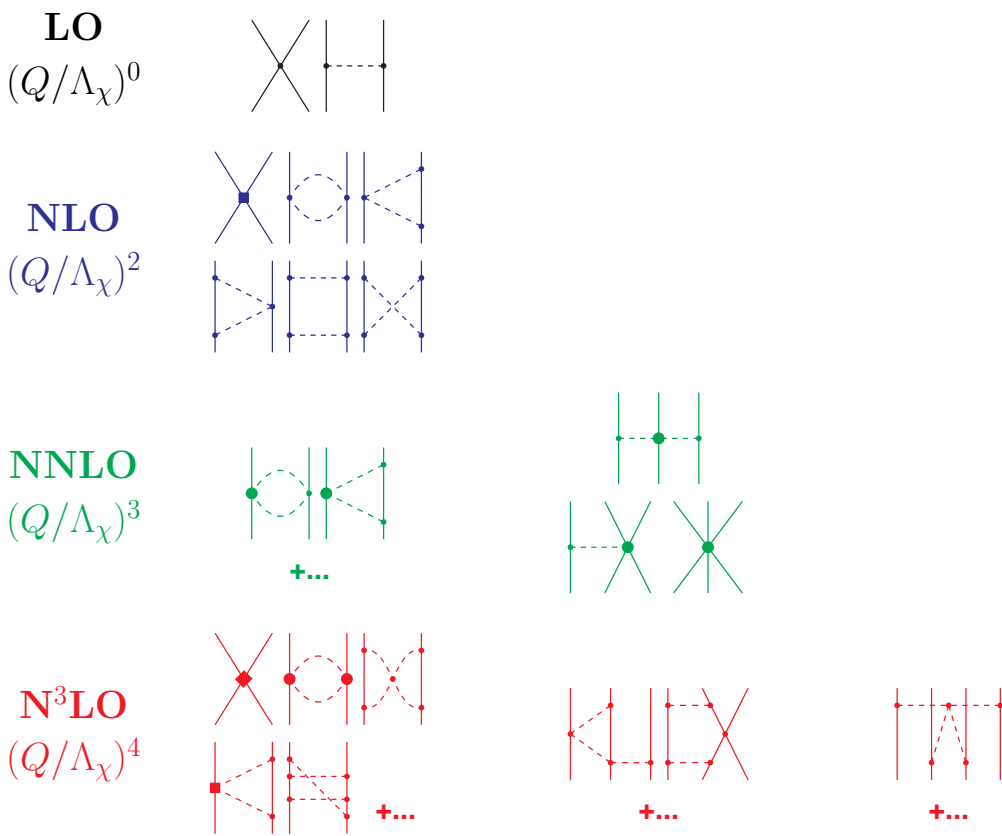

Figure 1. Hierarchy of nuclear forces in ChPT. Solid lines represent nucleons and dashed lines pions. Small dots, large solid dots, solid squares, and solid diamonds denote vertices of index $\Delta_{i}=0,1,2$, and 4, respectively. Further explanations are given in the text.

neutron matter [36-39] — with a great deal of success. The majority of nuclear structure calculations is nowadays based upon chiral forces.

However, in spite of this progress, we are not done. Due to the complexity of the nuclear force issue, there are still many subtle and not so subtle open problems. We will not list and discuss all of them, but instead just focus on the two open issues, which we perceive as the most important ones:

- The proper renormalization of chiral nuclear potentials and

- Subleading chiral few-nucleon forces.

Table 2. $\chi^{2}$ /datum for the reproduction of the $1999 n p$ database below $290 \mathrm{MeV}$ by various $n p$ potentials. $T_{\text {lab }}$ denotes the kinetic energy of the incident neutron in the laboratory system.

\begin{tabular}{cccccc}
\hline$T_{\text {lab }}$ bin $(\mathrm{MeV})$ & \# of $n p$ data & $\mathrm{N}^{3} \mathrm{LO}[12]$ & NNLO [15] & NLO [15] & AV18 [16] \\
\hline $0-100$ & 1058 & 1.05 & 1.7 & 4.5 & 0.95 \\
$100-190$ & 501 & 1.08 & 22 & 100 & 1.10 \\
$190-290$ & 843 & 1.15 & 47 & 180 & 1.11 \\
\hline $0-290$ & 2402 & 1.10 & 20 & 86 & 1.04 \\
\hline
\end{tabular}




\section{Renormalization of chiral nuclear forces}

The two-nucleon system is characterized by large scattering lengths and shallow (quasi) bound states which require a nonperturbative treatment. Following Weinberg's prescription [7], this is accomplished by inserting the potential $V$ into the Lippmann-Schwinger (LS) equation:

$$
T\left(\vec{p}^{\prime}, \vec{p}\right)=V\left(\vec{p}^{\prime}, \vec{p}\right)+\int d^{3} p^{\prime \prime} V\left(\vec{p}^{\prime}, \vec{p}^{\prime \prime}\right) \frac{M_{N}}{p^{2}-p^{\prime \prime 2}+i \epsilon} T\left(\vec{p}^{\prime \prime}, \vec{p}\right),
$$

where $M_{N}$ denotes the nucleon mass.

In general, the integral in the LS equation is divergent and needs to be regularized. One way to achieve this is by multiplying $V$ with a regulator function

$$
V\left(\vec{p}^{\prime}, \vec{p}\right) \longmapsto V\left(\vec{p}^{\prime}, \vec{p}\right) \boldsymbol{e}^{-\left(p^{\prime} / \Lambda\right)^{2 n}} \boldsymbol{e}^{-(p / \Lambda)^{2 n}}
$$

Typical choices for the parameters that appear in the regulator are $\Lambda \approx 0.5 \mathrm{GeV}<\Lambda_{\chi} \approx 1 \mathrm{GeV}$ and $n \geq 2$.

In field theories, divergent integrals are not uncommon and methods have been designed to deal with them. One regulates the integrals and then removes the dependence on the regularization parameters (scales, cutoffs) by "renormalization". In the end, the theory and its predictions do not depend on cutoffs or renormalization scales. So-called renormalizable quantum field theories, like QED, have essentially one set of prescriptions that takes care of renormalization through all orders. In contrast, EFTs are renormalized by "counter terms" (contact terms) that are introduced order by order in increasing numbers.

Naively, the most perfect renormalization procedure is the one where the cutoff parameter $\Lambda$ is taken to infinity while stable and quantitative results are maintained through the adjustment of counter terms. This was accomplished at LO in the work by Nogga et al [40]. At NNLO, the infinitecutoff renormalization procedure has been investigated in [41] for partial waves with total angular momentum $J \leq 1$ and in [42] for all partial waves with $J \leq 5$. However, for a quantitative chiral $N N$ potential one needs to advance all the way to $\mathrm{N}^{3} \mathrm{LO}$. At $\mathrm{N}^{3} \mathrm{LO}$, the ${ }^{1} S_{0}$ state was considered in Ref. [43], and all states up to $J=6$ were investigated in Ref. [44]. From all of these works, it is evident that no counter term is effective in partial-waves with short-range repulsion and only a single counter term can constructively be used in partial-waves with short-range attraction. Thus, for the $\Lambda \rightarrow \infty$ renormalization prescription, even at $\mathrm{N}^{3} \mathrm{LO}$, there exists either one or no counter term per partial-wave state. This is inconsistent with any reasonable power-counting scheme and prevents an order-by-order improvement of the predictions.

To summarize: In the infinite-cutoff renormalization scheme, the potential is admitted up to unlimited momenta. However, the EFT this potential is derived from has validity only for momenta smaller than the chiral symmetry breaking scale $\Lambda_{\chi} \approx 1 \mathrm{GeV}$. The lack of order-by-order convergence and discrepancies in lower partial-waves demonstrate that the potential should not be used beyond the limits of the effective theory [44] (see Ref. [45] for a related discussion). The conclusion then is that cutoffs should be limited to $\Lambda \lesssim \Lambda_{\chi}$ (but see also Ref. [46]).

Crucial for an EFT are regulator independence (within the range of validity of the EFT) and a power counting scheme that allows for order-by-order improvement with decreasing truncation error. The purpose of renormalization is to achieve this regulator independence while maintaining a functional power counting scheme.

Thus, in the spirit of Lepage [47], the cutoff independence should be examined for cutoffs below the hard scale and not beyond. Ranges of cutoff independence within the theoretical error are to be identified using Lepage plots [47]. Recently, we have started a systematic investigation of this kind. In 

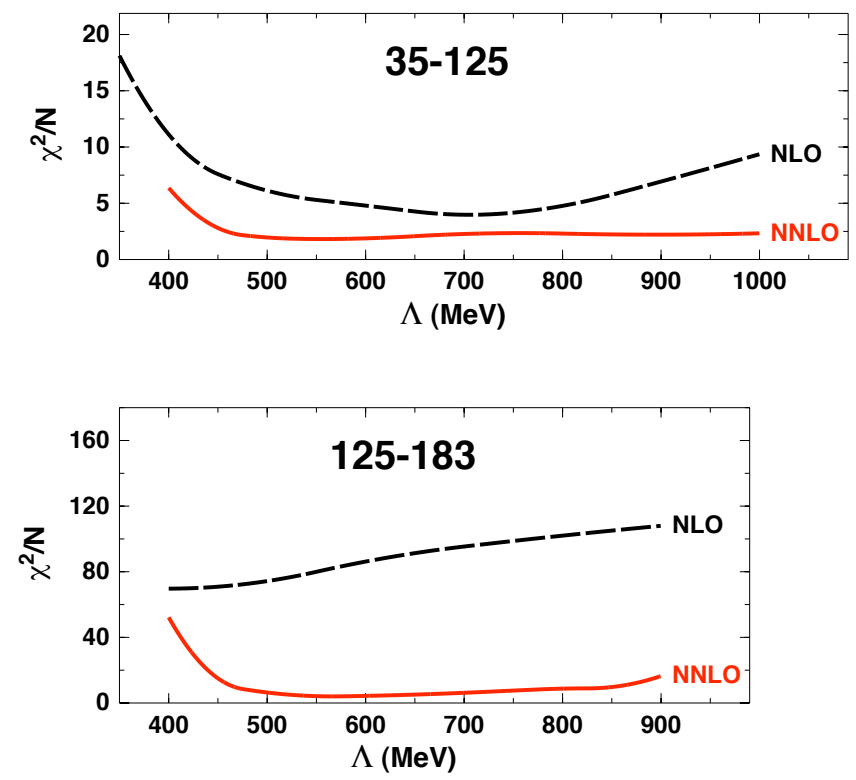

Figure 2. $\chi^{2} /$ datum for the reproduction of the $n p$ data in the energy range $35-125 \mathrm{MeV}$ (upper frame) and $125-183 \mathrm{MeV}$ (lower frame) as a function of the cutoff parameter $\Lambda$ of the regulator function Eq. (2). The (black) dashed curves show the $\chi^{2} /$ datum achieved with $n p$ potentials constructed at order NLO and the (red) solid curves are for NNLO.

our work, we quantify the error of the predictions by calculating the $\chi^{2} /$ datum for the reproduction of the neutron-proton $(n p)$ elastic scattering data as a function of the cutoff parameter $\Lambda$ of the regulator function Eq. (2). We have investigated the predictions by chiral $n p$ potentials at order NLO and NNLO applying Weinberg counting for the counter terms ( $N N$ contact terms). We show our results for the energy range 35-125 MeV in the upper frame of Fig. 2 and for 125-183 MeV in the lower frame. It is seen that the reproduction of the $n p$ data at these energies is generally poor at NLO, while at NNLO the $\chi^{2} /$ datum assumes acceptable values (a clear demonstration of order-by-order improvement). Moreover, at NNLO one observes "plateaus" of constant low $\chi^{2}$ for cutoff parameters ranging from 450 to $850 \mathrm{MeV}$. This may be perceived as cutoff independence (and, thus, successful renormalization) in the relevant range of cutoff parameters.

\section{Few-nucleon forces and what is missing}

We will now discuss the other issue we perceive as unfinished and important, namely, subleading chiral few-nucleon forces.

Nuclear three-body forces in ChPT were initially discussed by Weinberg [8]. The three-nucleon force (3NF) at NNLO, was derived by van Kolck [10] and applied, for the first time, in nucleondeuteron scattering by Epelbaum et al. [22]. The leading 4NF (at $\mathrm{N}^{3} \mathrm{LO}$ ) was constructed by Epelbaum [48] and found to contribute in the order of $0.1 \mathrm{MeV}$ to the ${ }^{4} \mathrm{He}$ binding energy (total ${ }^{4} \mathrm{He}$ binding energy: $28.3 \mathrm{MeV}$ ) in a preliminary calculation [49], confirming the traditional assumption that $4 \mathrm{NF}$ are essentially negligible. Therefore, the focus is on $3 \mathrm{NFs}$. 
For the order of a $3 \mathrm{NF}$, we have

$$
v=2+2 L+\sum_{i} \Delta_{i}
$$

where $L$ denotes the number of loops and $\Delta_{i}$ is the vertex index. We will use this equation to analyze $3 \mathrm{NF}$ contributions order by order. The first non-vanishing 3NF occurs at $v=3$ (NNLO), which is obtained when there are no loops $(L=0)$ and $\sum_{i} \Delta_{i}=1$, i.e., $\Delta_{i}=1$ for one vertex while $\Delta_{i}=0$ for all other vertices. There are three topologies which fulfill this condition, known as the two-pion exchange (2PE), one-pion exchange (1PE), and contact graphs (cf. Fig. 1).

The $3 \mathrm{NF}$ at NNLO has been applied in calculations of few-nucleon reactions [24], structure of light- and medium-mass nuclei [27-35], and nuclear and neutron matter [36-39] with a great deal of success. However, the famous ' $A_{y}$ puzzle' of nucleon-deuteron scattering [22] and the analogous problem with the analyzing power in $p-{ }^{3} \mathrm{He}$ scattering [26] is not resolved. Furthermore, the spectra of light nuclei leave room for improvement [28]. Since we are dealing with a perturbation theory, it is natural to turn to the next order when looking for improvements.

The next order is $\mathrm{N}^{3} \mathrm{LO}$, where we have loop and tree diagrams. For the loops, we have $L=1$ and, therefore, all $\Delta_{i}$ have to be zero to ensure $v=4$. Thus, these one-loop 3NF diagrams can include only leading order vertices, the parameters of which are fixed from $\pi N$ and $N N$ analysis. One sub-group of these diagrams (the 2PE graphs) has been calculated by Ishikawa and Robilotta [50], and the other topologies have been evaluated by the Bochum-Bonn group [51, 52]. The $\mathrm{N}^{3} \mathrm{LO} 2 \mathrm{PE}$ $3 \mathrm{NF}$ has been applied in the calculation of nucleon-deuteron observables in Ref. [50] causing little impact. Very recently, the long-range part of the chiral $\mathrm{N}^{3} \mathrm{LO} 3 \mathrm{NF}$ has been tested in the triton [53] and in three-nucleon scattering [54] yielding only moderate effects. The long- and short-range parts of this force have been used in neutron matter calculations (together with the $\mathrm{N}^{3} \mathrm{LO} 4 \mathrm{NF}$ ) producing relatively large contributions from the $3 \mathrm{NF}$ [55]. Thus, the ultimate assessment of the $\mathrm{N}^{3} \mathrm{LO} 3 \mathrm{NF}$ is still outstanding and will require more few- and many-body applications.

In the meantime, it is of interest to take already a look at the next order of $3 \mathrm{NFs}$, which is $\mathrm{N}^{4} \mathrm{LO}$ or $v=5$ (of the $\Delta$-less theory to which the present discussion is restricted because of lack of space). The loop contributions that occur at this order are obtained by replacing in the $\mathrm{N}^{3} \mathrm{LO}$ loops one vertex by a $\Delta_{i}=1$ vertex (with LEC $c_{i}$ ), which is why these loops may be more sizable than the $\mathrm{N}^{3} \mathrm{LO}$ loops. The 2PE topology turns out to be of modest size [56]; moreover, it can be handled in a practical way by summing it up together with the $2 \mathrm{PE}$ topologies at NNLO and $\mathrm{N}^{3} \mathrm{LO}$ [56]. The 2PE-1PE and ring topologies have also been evaluated [57]. Finally, a tree topology at $\mathrm{N}^{4} \mathrm{LO}$ provides a new set of $3 \mathrm{~N}$ contact interactions, which have recently been derived by the Pisa group [58]. Contact terms are typically simple (as compared to loop diagrams) and their coefficients are unconstrained (except for naturalness). Therefore, it would be an attractive project to test some terms (in particular, the spinorbit terms) of the $\mathrm{N}^{4} \mathrm{LO}$ contact $3 \mathrm{NF}$ [58] in calculations of few-body reactions (specifically, the p-d and $\mathrm{p}-{ }^{3} \mathrm{He} A_{y}$ ) and spectra of light nuclei.

\section{Conclusions and outlook}

The past 15 years have seen great progress in our understanding of nuclear forces in terms of lowenergy QCD. Key to this development was the realization that low-energy QCD is equivalent to an effective field theory which allows for a perturbative expansion that has become known as chiral perturbation theory. In this framework, two- and many-body forces emerge on an equal footing and the empirical fact that nuclear many-body forces are substantially weaker then the two-nucleon force is explained automatically. 
In spite of the great progress and success of the past 15 years, there are still some unresolved issues. One problem is the proper renormalization of the chiral two- and many-nucleon potentials, where systematic investigations are already under way (cf. Sec. 2).

The other unfinished business are the few-nucleon forces beyond NNLO (sub-leading few-nucleon forces) which are needed to hopefully resolve some important outstanding nuclear structure problems. At orders $\mathrm{N}^{3} \mathrm{LO}$ and $\mathrm{N}^{4} \mathrm{LO}$ very many new $3 \mathrm{NF}$ structures appear, some of which have already been tested. However, in view of the multitude of $3 \mathrm{NF}$ topologies it will take a while until we will have a proper overview of impact and convergence of these contributions.

If the open issues discussed in this paper will be resolved within the next few years, then, after 80 years of desperate struggle, we may finally claim that the nuclear force problem is essentially under control. The greatest beneficiaries of such progress will be the fields of exact few-nucleon calculations and $a b$ initio nuclear structure physics.

This work was supported in part by the U.S. Department of Energy under Grant No. DE-FG0203ER41270.

\section{References}

[1] H. Yukawa, Proc. Phys. Math. Soc. Japan 17, 48 (1935).

[2] Prog. Theor. Phys. (Kyoto), Supplement 3 (1956).

[3] M. Taketani, S. Machida, S. Onuma, Prog. Theor. Phys. (Kyoto) 7, 45 (1952).

[4] R. Machleidt, Adv. Nucl. Phys. 19, 189 (1989).

[5] R. Machleidt, K. Holinde, and Ch. Elster, Phys. Rep. 149, 1 (1987).

[6] S. Weinberg, Physica 96A, 327 (1979).

[7] S. Weinberg, Nucl. Phys. B363, 3 (1991).

[8] S. Weinberg, Phys. Lett. B 295, 114 (1992).

[9] C. Ordóñez, L. Ray, U. van Kolck, Phys. Rev. Lett. 72, 1982 (1994).

[10] U. van Kolck, Phys. Rev. C 49, 2932 (1994).

[11] N. Kaiser, R. Brockmann, and W. Weise, Nucl. Phys. A625, 758 (1997).

[12] D. R. Entem, R. Machleidt, Phys. Rev. C 68, 041001 (2003).

[13] E. Epelbaum, W. Glöckle, and U.-G. Meißner, Nucl. Phys. A747, 362 (2005).

[14] R. Machleidt and D. R. Entem, Phys. Rep. 503, 1 (2011), and references therein.

[15] E. Epelbaum, W. Glöckle, and U.-G. Meißner, Eur. Phys. J. A19, 401 (2004).

[16] R. B. Wiringa, V. G. J. Stoks, and R. Schiavilla, Phys. Rev. C 51, 38 (1995).

[17] A. Deltuva and A. C. Fonseca, Phys. Rev. Lett. 98, 162502 (2007); Phys. Rev. C 76, 021001 (2007); arXiv:1005.1308 [nucl-th].

[18] L. Coraggio, A. Covello, A. Gargano, and N. Itaco, Phys. Rev. C 81, 064303 (2010).

[19] P. Navrátil and E. Caurier, Phys. Rev. C 69, 014311 (2004).

[20] G. Hagen, T. Papenbrock, D. J. Dean, and M. Hjorth-Jensen, Phys. Rev. Lett. 101, 092502 (2008).

[21] S. Fujii, R. Okamoto, and K. Suzuki, Phys. Rev. Lett. 103, 182501 (2009).

[22] E. Epelbaum, A. Nogga, W. Glöckle, H. Kamada, U.-G. Meißner, and H. Witala, Phys. Rev. C 66, 064001 (2002).

[23] H. Witala et al., Phys. Rev. C 73, 044004 (2006).

[24] N. Kalantar-Nayestanaki, E. Epelbaum, E. Messchendorp, and A. Nogga, Rep. Prog. Phys. 75, 016301 (2012). 
[25] A. Kievsky, M. Viviani, L. Girlanda, and L. E. Marcucci, Phys. Rev. C 81, 044003 (2010).

[26] M. Viviani, L. Giarlanda, A. Kievsky, L. E. Marcucci, and S. Rosati, arXiv:1004.1306 [nucl-th].

[27] A. Nogga, P. Navratil, B. R. Barrett, and J. P. Vary, Phys. Rev. C 73, 064002 (2006).

[28] P. Navratil, V. G. Gueorguiev, J. P. Vary, W. E. Ormand, and A. Nogga, Phys. Rev. Lett. 99, 042501 (2007).

[29] T. Otsuka, T. Susuki, J. D. Holt, A. Schwenk, and Y. Akaishi, Phys. Rev. Lett. 105, 03250 (2010).

[30] J. W. Holt, N. Kaiser, and W. Weise, Phys. Rev. C 79, 054331 (2009).

[31] R. Roth, S. Binder, K. Vobig, A. Calci, J. Langhammer, and P. Navratil, Phys. Rev. Lett. 109, 052501 (2012).

[32] H. Hergert, S. K. Bogner, S. Binder, A. Calci, J. Langhammer, R. Roth and A. Schwenk, Phys. Rev. C 87, 034307 (2013).

[33] G. Hagen, M. Hjorth-Jensen, G. R. Jansen, R. Machleidt, and T. Papenbrock, Phys. Rev. Lett. 108, 242501 (2012).

[34] G. Hagen, M. Hjorth-Jensen, G. R. Jansen, R. Machleidt, and T. Papenbrock, Phys. Rev. Lett. 109, 032502 (2012).

[35] A. Ekström et al., Phys. Rev. Lett. 110, 192502 (2013).

[36] K. Hebeler and A. Schwenk, Phys. Rev. C 82, 014314 (2010).

[37] K. Hebeler, S.K. Bogner, R. J. Furnstahl, A. Nogga, and A. Schwenk, Phys. Rev. C 83, 031301(R) (2011).

[38] F. Sammarruca, B. Chen, L. Coraggio, N. Itaco, and R. Machleidt, Phys. Rev. C 86, 054317 (2013).

[39] L. Coraggio, J. W. Holt, N. Itaco, R. Machleidt, and F. Sammarruca, Phys. Rev. C 87, 014322 (2013).

[40] A. Nogga, R. G. E. Timmermans, and U. van Kolck, Phys. Rev. C 72, 054006 (2005).

[41] C.-J. Yang, Ch. Elster, D. R. Phillips, Phys. Rev. C 80, 044002 (2009).

[42] M. Pavon Valderrama, and E. Ruiz Arriola, Phys. Rev. C 74, 064004 (2006).

[43] D. R. Entem, E. Ruiz Arriola, M. Pavón Valderrama, and R. Machleidt, Phys. Rev. C 77, 044006 (2008).

[44] Ch. Zeoli, R. Machleidt, D. R. Entem, Few-Body Syst. DOI 10.1007/s00601-012-0481-4, arXiv:1208.2657 [nucl-th].

[45] E. Epelbaum and J. Gegelia, Eur. Phys. J. A41, 341 (2009).

[46] E. Epelbaum and J. Gegelia, Phys. Lett B 716, 338 (2012).

[47] G. P. Lepage, How to Renormalize the Schrödinger Equation, arXiv:nucl-th/9706029.

[48] E. Epelbaum, Eur. Phys. J. A34, 197 (2007).

[49] D. Rozpedzik et al., Acta Phys. Polon. B37, 2889 (2006).

[50] S. Ishikawa and M. R. Robilotta, Phys. Rev. C 76, 014006 (2007).

[51] V. Bernard, E. Epelbaum, H. Krebs, and U.-G. Meißner, Phys. Rev. C 77, 064004 (2008).

[52] V. Bernard, E. Epelbaum, H. Krebs, and U.-G. Meißner, Phys. Rev. C 84, 054001 (2011).

[53] R. Skibinski et al., Phys. Rev. C 84, 054005 (2011).

[54] H. Witała, privat communication.

[55] I. Tews, T. Krüger, K. Hebeler, and A. Schwenk, Phys. Rev. Lett. 110, 032504 (2013).

[56] H. Krebs, A. Gasparyan, and E. Epelbaum, Phys. Rev. C 85, 054006 (2012).

[57] H. Krebs, A. Gasparyan, and E. Epelbaum, Phys. Rev. C 87, 054007 (2013).

[58] L. Girlanda, A. Kievsky, M. Viviani, Phys. Rev. C 84, 014001 (2011). 